\title{
Gaze vaselinada para prevenção de lesões por pressão em pessoas com dermatoses imunobolhosas
}

\author{
Vaseline gauze for prevention of pressure injuries in people with immunobullous dermatoses \\ Gasa con vaselina para la prevención de lesiones por presión en personas con dermatosis inmunobulosa
}

\author{
Euzeli da Silva Brandão'; Iraci dos Santos"'; Regina Serrão Lanzillottill', Marilange Aparecida Sampaio RossilV
}

\begin{abstract}
RESUMO
Objetivo: avaliar a mobilidade do cliente com dermatose imunobolhosa antes e após aplicação do curativo com gaze vaselinada. Método: estudo quase experimental, interinstitucional, com clientes com dermatoses imunobolhosas hospitalizados em um hospital estadual e um hospital federal do Estado do Rio de Janeiro e uma instituição do Mato Grosso do Sul. Utilizou-se a lógica fuzzy para classificar a mobilidade dos sujeitos antes, 24 horas após e uma semana após aplicação do curativo. A pesquisa foi aprovada pelo Comitê de Ética em Pesquisa. Resultados: Incluídos 14 participantes, sendo nove com pênfigo vulgar, dois com pênfigo foliáceo e três com penfigóide bolhoso, entre 27 e 82 anos, predominando 11 mulheres. Após 24 horas, nenhum participante se considerou com baixa mobilidade, sete passaram a mobilidade média, e sete, alta, o que foi mantido uma semana após aplicação do curativo. Conclusão: constatou-se significativo aumento da mobilidade logo nas primeiras 24 horas após aplicação do curativo.
\end{abstract}

Descritores: Cuidados de Enfermagem; Prevenção Secundária; Pênfigo; Dermatopatias; Lesão por Pressão.

\begin{abstract}
Objective: to assess the mobility of clients with immunobullous dermatoses, before and after applying vaseline gauze dressings. Method: in this quasi-experimental, interinstitutional study of inpatients with immunobullous dermatoses at a state hospital and a federal hospital in Rio de Janeiro State and an institution in Mato Grosso do Sul (Brazil), patient mobility before, 24 hours after, and one week after applying the dressing was classified using fuzzy logic. The study was approved by the research ethics committee. Results: 14 participants, nine with pemphigus vulgaris, two with pemphigus foliaceus, and three with bullous pemphigoid, aged between 27 and 82 years old, and predominantly (11) women. After 24 hours, none of the participants considered their mobility to be poor, seven began to be moderately mobile, and seven were highly mobile, and continued so one week after applying the dressing. Conclusion: mobility increased significant in the first 24 hours after applying the dressing. Descriptors: Nursing Care; Secondary Prevention; Pemphigus; Skin Diseases; Pressure Ulcer.
\end{abstract}

\section{RESUMEN}

Objetivo: evaluar la movilidad de clientes con dermatosis inmunobullosa, antes y después de la aplicación de apósitos de gasa con vaselina. Método: en este estudio cuasi-experimental, interinstitucional de pacientes hospitalizados con dermatosis inmunobullosa en un hospital estatal y un hospital federal en el estado de Río de Janeiro y una institución en Mato Grosso do Sul (Brazil), la movilidad del paciente antes, 24 horas después y una semana después la aplicación del apósito se clasificó mediante lógica difusa. El estudio fue aprobado por el comité de ética en investigación. Resultados: se incluyeron 14 participantes, nueve con pénfigo vulgar, dos con pénfigo foliáceo y tres con penfigoide ampolloso, con edades comprendidas entre 27 y 82 años, y predominantemente mujeres $(n=11)$. Después de 24 horas, ninguno de los participantes consideró que su movilidad fuera pobre, siete comenzaron a ser moderadamente móviles y siete eran altamente móviles, y así continuaron una semana después de la aplicación del apósito. Conclusión: la movilidad aumentó significativamente en las primeras 24 horas después de la aplicación del apósitoconsideraba con baja movilidad, siete comenzaron a tener movilidad media y siete, alta, que se mantuvo una semana después de aplicar el apósito. Conclusión: hubo un aumento significativo en la movilidad en las primeras 24 horas después de aplicar el apósito.

Descriptores: Cuidados de Enfermería; Prevencíon Secundaria; Pénfigo; Enfermedades de la Piel; Úlcera por Presión.

\section{INTRODUÇÃO}

As dermatoses imunobolhosas (DI) são raras, de evolução crônica cuja manifestação consiste no desenvolvimento de bolhas e vesículas na pele e/ou mucosas ${ }^{1}$.

Tais afeç̧ões cutâneas normalmente dão origem a lesões extensas e disseminadas, causando dor e desconforto, tornando o cuidar em enfermagem um desafio, pela sua complexidade e vulnerabilidade a complicações ${ }^{2}$.

\footnotetext{
'Enfermeira. Doutora. Professora Adjunta. Universidade Federal Fluminense. Niterói, Rio de Janeiro, Brasil. E-mail: euzelibrandao@gmail.com. ORCID: http://orcid.org/0000-0001-8988-8103

"Enfermeira. Doutora. Professora Titular. Universidade do Estado do Rio de Janeiro, Rio de Janeiro, Brasil. E-mail: iraci.s@terra.com.br. ORCID: http://orcid.org/00000002-1734-5561

I'Estatística. Doutora. Professora Adjunta. Universidade do Estado do Rio de Janeiro, Rio de Janeiro, Brasil. E-mail: reginalanzillotti@gmail.com. ORCID:

http://orcid.org/0000-0001-7789-6843

IVEnfermeira. E-mail: marilange.rossi@bol.com.br. ORCID: http://orcid.org/0000-0001-5737-372X
} 
Neste contexto, destaca-se que as lesões decorrentes das DI podem interferir na vida da pessoa nas esferas clínica, social e emocional. Entre as limitações destaca-se a redução da mobilidade, aumentando a sua vulnerabilidade para o desenvolvimento de outras lesões, em especial, as lesões por pressão (LP), tendo em vista a existência de fatores intrínsecos simultâneos como falta de integridade da pele preexistente, fragilidade da epiderme, uso de corticóide em altas doses, desnutrição, lesões em cavidade oral, dificuldade de mobilização/imobilidade, principalmente nos casos de lesões disseminadas e mantidas expostas.

Destaca-se que, por se tratar de doença rara, as pesquisas existentes normalmente limitam-se a análises epidemiológicas e técnicas de manejo farmacológico, sem privilegiar outros aspectos que envolvem o atendimento a esta clientela específica, em especial, no campo das políticas públicas de saúde ${ }^{3}$. Diante da precariedade de referenciais teóricos relacionados ao cuidado de enfermagem aos clientes com $\mathrm{DI}^{4}$, inclusive com níveis de evidência significativos em relação aos curativos $^{5}$, foi proposto um protocolo de curativo com gaze vaselinada, na tentativa de melhorar a mobilidade, promover o conforto e minimizar complicações, incluindo as LP.

As DI decorrem da ativação do sistema imunológico contra constituintes próprios do organismo, neste caso, contra estruturas específicas da pele, consideradas autoantígenos localizados nas regiões intraepidérmica ou subepidérmica, sendo indispensável à identificação de sua localização para classificação do tipo de $\mathrm{DI}^{1}$.

Os pênfigos são DI intraepidérmicas, por isso, as lesões bolhosas são mais superficiais e sensíveis, rompendo-se com mais facilidade que as de origem subepidérmicas. Existem diferentes tipos de pênfigo: vulgar (PV), foliáceo (PF), induzido por drogas, herpetiforme, paraneoplásico e por imunoglobulina A (IgA), sendo os dois primeiros considerados principais $^{1}$.

Apesar da etiologia desconhecida dos pênfigos, há referências de que a base do processo autoimune seriam as moléculas de adesão intercelular, denominadas caderinas, localizadas nos desmossomos. Estas agem como placas de aderência unindo os ceratinócitos, sendo estruturas visíveis apenas por microscopia eletrônica. Por motivo desconhecido, os desmossomos se tornam antigênicos, estimulando a produção de anticorpos, provocando a perda de adesão entre os ceratinócitos, processo chamado de acantólise ${ }^{1}$.

Entre as DI subepidérmicas que compõem o complexo penfigóide estão o penfigóide bolhoso (PB), o penfigóide das membranas mucosas, o penfigóide gestacional, a dermatite herpetiforme, a dermatose por IgA linear e a epidermólise bolhosa adquirida ${ }^{1}$.

Embora o indivíduo com diagnóstico médico de DI possa apresentar áreas de pele sadia, ressalta-se que toda a epiderme está vulnerável. Assim, qualquer pressão na pele pode promover o desenvolvimento de lesões, em especial as LP.

A LP caracteriza-se por um dano na pele e/ou tecidos moles subjacentes, geralmente sobre uma proeminência óssea ou relacionada ao uso de dispositivo médico/outro. Esta ocorre como um resultado da pressão intensa e/ou prolongada combinada com cisalhamento. A tolerância do tecido mole à pressão e ao cisalhamento também pode ser afetada por outros fatores como microclima, nutrição, perfusão, comorbidades e condição do tecido ${ }^{6}$, fato que predispõe ainda mais as pessoas com $\mathrm{Dl}$ ao desenvolvimento dessas lesões.

Destaca-se que embora as DI possuam diferenças na localização da acantólise (intraepidérmica ou subepidérmica), as características clínicas, em especial, em relação as lesões bolhosas disseminadas normalmente apresentadas pela pessoa acometida afetam o conforto e a mobilidade de forma semelhante, facilitando o desenvolvimento de LP.

Assim, levantou-se como objetivo avaliar a mobilidade do cliente com dermatose imunbolhosa antes e após aplicação do curativo com gaze vaselinada.

\section{MÉTODO}

Estudo quase experimental, interinstitucional, realizado de junho/2012 a abril/2013, com clientes com dermatoses imunobolhosas hospitalizados em um hospital estadual e um hospital federal do Estado do Rio de Janeiro, além de uma instituição do Mato Grosso do Sul. Os campos de pesquisas foram selecionados considerando a possibilidade de contemplar instituições que possuem enfermarias de dermatologia, localizadas em diferentes estados brasileiros, ou que possuem leitos especificamente destinados a esta clientela.

O estudo quase experimental foi escolhido, considerando que se trata de enfermidades raras. Assim, utilizou-se delineamento alternativo destinado a um único grupo não randomizado. Como não existe grupo controle, em cada sujeito, considerou-se o seu próprio controle, avaliado antes e após intervenção. A seleção dos participantes foi realizada segundo amostragem por conveniência, nela incluíram-se os que atenderam aos seguintes critérios de inclusão: adultos com diagnóstico definitivo de DI em atividade, hospitalizados nas enfermarias das instituições citadas, no período determinado para realização da pesquisa, independente de histórico de internações prévias, sexo, faixa etária e uso ou não de medicação sistêmica. Foram estabelecidos os seguintes critérios de exclusão: pessoas em tratamento psiquiátrico, desorientadas no tempo e no espaço. 
Importa mencionar que os participantes foram avaliados de forma integral, mediante utilização do "Protocolo para avaliação do cliente com afecções cutâneas", validado por especialistas ${ }^{7}$, sendo identificadas, através deste, as variáveis sociodemográficas e clínicas: idade, sexo, faixa etária, tipo de DI, índice de comprometimento cutâneo mucoso, além da presença ou não dos diagnósticos de enfermagem mobilidade física prejudicada e mobilidade no leito prejudicada, e suas respectivas características definidoras e/ou fatores relacionados, conforme a NANDA-I ${ }^{8}$. Nos casos dos clientes com PV, a avaliação da extensão das lesões cutâneas e mucosas foi realizada por um instrumento validado, o índice de comprometimento cutâneo-mucoso do pênfigo vulgar (ICCMPV) ${ }^{9}$. Considerou-se variável independente, o curativo proposto e dependente, a alteração no padrão de mobilidade.

Após avaliação, foram realizadas as aferições da mobilidade junto aos participantes, nos momentos antes ( $\left.T_{0}\right), 24$ horas após $\left(\mathrm{T}_{1}\right)$ e uma semana após $\left(\mathrm{T}_{2}\right)$ aplicação do curativo. As proposições direcionadas aos participantes para avaliação da mobilidade tiveram as respostas indicadas em uma escala cromática ascendente, iniciada pela cor branca, seguida por tonalidades de amarelo, laranja e vermelho, correspondendo ao suporte dos conjuntos fuzzy. Estes conjuntos foram tratados por Loti Zadeh, pesquisador que quebrou as fronteiras do conhecimento pela Lógica Fuzzy ${ }^{10}$.

Para permitir a visualização gráfica das variáveis linguísticas fuzzy ("Baixa" "Média" e "Alta”), optou-se pela utilização de trapézios com suporte constituído pela escala cromática, conforme demonstrado na Figura 1 . Cada participante expressou a percepção da sua mobilidade em função da cor nos três momentos. $O$ aumento na tonalidade da cor significava maior intensidade, e a redução, menor. A distribuição de frequência dos participantes em relação a mobilidade nos momentos $\mathrm{T}_{0}, \mathrm{~T}_{1}$ e $\mathrm{T}_{2}$ permitiu realizar uma análise estatística descritiva em relação aos termos linguísticos (baixa, média e alta) com as respectivas pertinências.

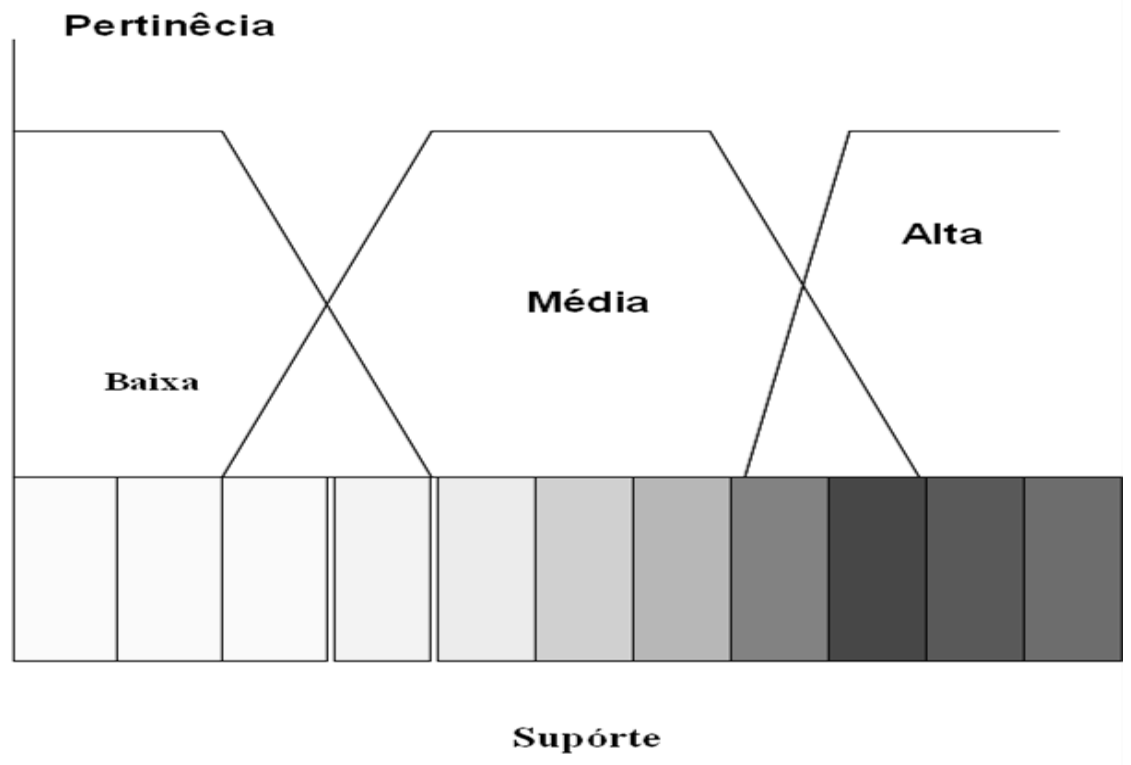

FIGURA 1: Conjuntos fuzzy para avaliação da mobilidade e respectivas pertinências nos momentos $\mathrm{T}_{0}, \mathrm{~T}_{1}$ e $\mathrm{T}_{2}$.

Nota: Suporte dos conjuntos fuzzy (escala cromática) iniciada pela cor branca, seguida por tonalidades de amarelo, laranja e vermelho.

Assim, o valor de pertinência foi obtido mediante os conjuntos fuzzy, variáveis linguísticas fuzzy. Cada escala cromática com as respectivas marcações realizadas pelos participantes referentes a sua mobilidade foi superposta ao suporte cromático do gráfico. O reconhecimento de padrões foi traduzido de acordo com a classificação dos termos linguísticos "Baixa", "Média" e "Alta" com as respectivas pertinências em cada um dos três momentos To, $T_{1}$ e $T_{2}$. 
Os valores relativos às pertinências devem ser compreendidos como uma escala de valoração crescente no intervalo entre zero e um ${ }^{10}$. Exemplificando, se dois clientes forem classificados com o termo linguístico "baixo" para o atributo mobilidade, deve-se observar a pertinência atribuída a cada um deles, pois aquele que obtiver menor grau de pertinência deve ser considerado em situação de menor mobilidade.

A aplicação do curativo com gaze vaselinada ocorreu logo após a avaliação dos participantes e aferição de sua mobilidade no momento $\left(\mathrm{T}_{0}\right)$. Destaca-se que os curativos diários foram preparados e realizados por enfermeiros especialistas em dermatologia e/ou capacitados para realizar o procedimento, inclusive no Hospital Adventista do Pênfigo/MS. Sobre isso, importa mencionar que o curativo seguiu um protocolo, visando padronização do seu preparo, além de testes de esterilidade realizados no Laboratório de Bacteriologia Clínica (LABAC) e CONTQUAL - Laboratório de Controle de qualidade do Hospital Universitário Pedro Ernesto/UERJ, comprovando a garantia da esterilização.

O preparo do curativo foi realizado com os seguintes materiais: gaze em rolo com $10 \mathrm{~cm}$ de largura, pote de vaselina sólida, vaselina líquida, caixa de aço inoxidável com dimensões mínimas 35×18x07cm, etiqueta, invólucro de tamanho superior a caixa para esterilização em autoclave. Etapas: cortar 10 faixas de gaze rolo ( $35 \mathrm{~cm}$ de comprimento). Umedecer cada faixa com $20 \mathrm{ml}$ de vaselina líquida e depois impregnar com $35 \mathrm{~g}$ de vaselina sólida. Colocar duas faixas no fundo da caixa, lado a lado, sobrepondo às demais até no máximo cinco de cada lado, totalizando 10 faixas. (o limite preconizado de $1 / 3$ de altura de gaze na caixa viabiliza a esterilização, evita transbordamento da vaselina e contaminação). Envolver totalmente a caixa com gaze rolo aberta na largura. Colocar etiqueta de identificação (setor, especificação do material, data do preparo e nome do profissional). Fixar a gaze e etiqueta com fita adesiva. Envolver a caixa com invólucro específico para esterilização em autoclave. Encaminhar para esterilização em autoclave.

Declara-se que foi adquirido registro de patente deste curativo pelo Instituto Nacional da Propriedade Industrial, número do processo BR 1020160241421.

A aplicação do curativo foi realizada após higiene corporal e limpeza das lesões com soro fisiológico aquecido a 36 em jato; secagem suave somente das regiões de pele íntegra com compressa estéril; aplicação do curativo com gaze vaselinada em todas as lesões utilizando técnica asséptica; proteção com compressa estéril e atadura de crepom ou malha tubular, fixando com fita adesiva, sem pressionar. O curativo deverá ser trocado diariamente após banho de aspersão.

O projeto da pesquisa foi cadastrado na Plataforma Brasil e submetido à avaliação, sendo aprovado conforme protocolo 0258.0.228.000-11.

Ressalte-se apoio financeiro, pelo CNPq - Ministério da Ciência, Tecnologia e Inovação, para seu desenvolvimento no período de 2012 a 2014, através da submissão ao Edital Universal 14/2011-Faixa B- Processo: 477063/2011-0.

\section{RESULTADOS}

Participaram do estudo 14 pessoas, sendo 11 clientes com pênfigo (nove vulgar e dois foliáceo) e três PB. A faixa etária entre 27 e 82 anos, predominando 11 mulheres. No que diz respeito à avaliação do ICCMPV, entre os nove participantes com esta doença, três apresentaram índice 60, dois 80 e quatro 30, 35, 40 e 100, revelando que seis apresentaram índice bastante expressivo, acima de 60. A extensão das lesões dos dois participantes com PF e os três com PB foram consideradas extensas, pois atingiam face, tronco e membros superiores e inferiores, apenas um com PB, apresentava lesões bolhosas nas mãos e pés.

No que tange a mobilidade dos participantes, ressalta-se que no momento $T_{0}$, sete indicaram baixa mobilidade e cinco, média mobilidade, como apresentado na Figura 2, fato que revela a interferência das lesões decorrentes das DI na mobilização do cliente. Assim, durante a avaliação dos participantes do estudo foi possível constatar a vulnerabilidade dos mesmos as LP, não somente devido ao prévio comprometimento da integridade da pele em decorrência das lesões decorrentes das DI; mas também, a presença dos diagnósticos de enfermagem mobilidade física e no leito prejudicada.

No momento $T_{1}$, após o primeiro dia da aplicação do curativo, nenhum cliente se considerou com baixa mobilidade, pois sete passaram a apresentar mobilidade média, e os sete restantes, mobilidade alta, o que foi mantido no momento $T_{2}$. Esclarecendo-se que um dos clientes não foi avaliado no momento $T_{2}$ devido solicitação de alta para continuidade do tratamento em instituição privada. Assim, percebeu-se que, a mudança mais expressiva na condição do cliente em relação à sua mobilidade, ocorreu nas primeiras 24 horas de intervenção.

A análise personalizada dos clientes, apesentada na Tabela 1, revela que na transição do momento $T_{0}$ para o $T_{1}$, nove participantes consideraram aumento da mobilidade, e cinco mantiveram-se na mesma condição, fato que revela um índice de performance positiva de $64,29 \%$. Do momento $T_{1}$ para $T_{2}$, apenas três participantes dos treze avaliados referiram aumento em sua mobilidade, índice de desempenho de 23,08\%. Ainda, seis mantiveram-se na mesma condição, com índice de 
estacionalidade de $46,15 \%$, e quatro consideraram que houve redução de alta para média mobilidade, com índice de insatisfação de $30,77 \%$. No último momento, a totalidade dos sujeitos caiu para treze, devido à alta solicitada.

\section{Sujeitos segundo mobilidade}

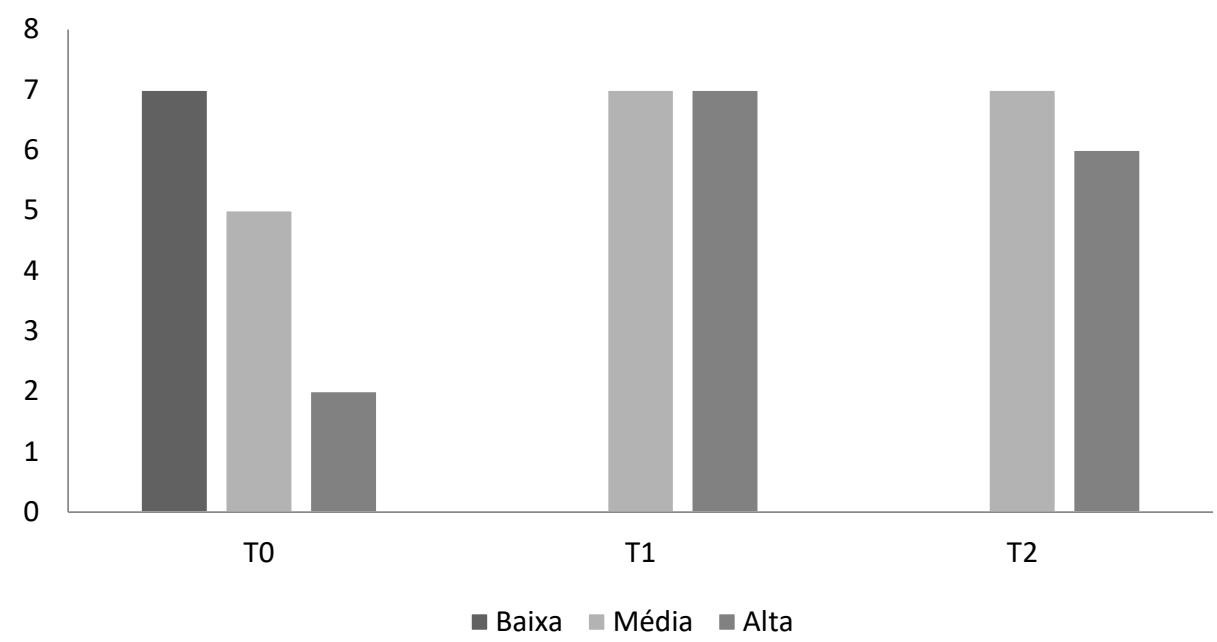

FIGURA 2: Distribuição dos sujeitos segundo os termos linguísticos em relação ao atributo mobilidade nos momentos $T_{0}, T_{1}$ e $T_{2}$. Rio de Janeiro, Rio de Janeiro, Brasil. 2013.

TABELA 1: Evolução dos sujeitos segundo os termos linguísticos em relação a mobilidade nos momentos $T_{0}, T_{1}$ e $T_{2}$ com respectivas pertinências. Rio de Janeiro, Rio de Janeiro, Brasil, 2013.

\begin{tabular}{|c|c|c|c|c|c|c|c|c|}
\hline \multirow{3}{*}{ SUJEITOS } & \multirow{2}{*}{\multicolumn{2}{|c|}{ Momento T0 }} & \multirow{2}{*}{\multicolumn{2}{|c|}{ Momento T1 }} & \multirow{2}{*}{\multicolumn{2}{|c|}{ Momento T2 }} & \multirow{2}{*}{\multicolumn{2}{|c|}{ EVOLUÇÃO }} \\
\hline & & & & & & & & \\
\hline & CLASS & PERT & CLASS & PERT & CLASS & PERT & $\mathrm{T}_{0}$ a $\mathrm{T}_{1}$ & $\mathrm{~T}_{1}$ a $\mathrm{T}_{2}$ \\
\hline 1 & Média & 0,70 & Média & 1,00 & Alta & 0,15 & Manutenção & Aumento \\
\hline 2 & Média & 1,00 & Alta & 1,00 & Alta & 1,00 & Aumento & Manutenção \\
\hline 3 & Baixa & 1,00 & Média & 0,80 & Média & 0,25 & Aumento & Manutenção \\
\hline 4 & Baixa & 1,00 & Alta & 0,70 & Média & 0,25 & Aumento & Redução \\
\hline 5 & Baixa & 1,00 & Média & 1,00 & Média & 1,00 & Aumento & Manutenção \\
\hline 6 & Baixa & 1,00 & Alta & 0,70 & Média & 0,25 & Aumento & Redução \\
\hline 7 & Média & 0,75 & Média & 1,00 & - & - & Manutenção & - \\
\hline 8 & Média & 1,00 & Alta & 0,75 & Alta & 0,25 & Aumento & Manutenção \\
\hline 9 & Baixa & 1,00 & Média & 1,00 & Alta & 0,20 & Aumento & Aumento \\
\hline 10 & Baixa & 0,85 & Alta & 0,55 & Média & 1,00 & Aumento & Redução \\
\hline 11 & Alta & 0,75 & Alta & 1,00 & Alta & 0,25 & Manutenção & Manutenção \\
\hline 12 & Média & 1,00 & Média & 1,00 & Média & 1,00 & Manutenção & Manutenção \\
\hline 13 & Alta & 0,70 & Alta & 0,70 & Média & 1,00 & Manutenção & Redução \\
\hline 14 & Baixa & 1,00 & Média & 1,00 & Alta & 0,00 & Aumento & Aumento \\
\hline
\end{tabular}

Legenda: CLASS: Classificação; PERT: Pertinência.

Nota: O cliente 7 permaneceu internado por quatro dias, fato que impossibilitou a avaliação no momento $\mathrm{T}_{2}$. Assim no momento $\mathrm{T}_{2}$, totalizaram 13 clientes.

No momento $T_{0}$, os clientes 3,4,5,6,9,10 e 14 foram classificados com mobilidade Baixa com pertinências máximas para a maioria, excetuando-se o cliente 10 ao qual foi atribuído grau de pertinência 0,85 . No momento $T_{1}$, ocorreu migração para a classificação Alta, dos sujeitos 4, 6 e 10, aferidos com pertinências 0,70 para os dois primeiros e 0,55 para o outro. Os demais foram categorizados em mobilidade Média, tendo pertinências 1,00, excluindo-se o cliente 3, valor 0,80. Na terceira aferição, apenas os clientes 9 e 14 migraram para a classe Alta com pertinências muito baixa, 0,2 e nula, respectivamente. 
Os participantes 1, 7 e 12 foram classificados com mobilidade Média na primeira migração com pertinências de 0,70 para 1,0 , de 0,75 para 1,0 e retenção em 1.0 , respectivamente, o que mostra uma ascensão de melhora na mesma categorização. Na segunda transição, mais uma melhora, pois um deles transferiu-se para a classificação Alta com pertinência 0,15.

Conforme demonstrado, os clientes 11 e 13 foram avaliados na classe de mobilidade Alta, no primeiro momento, com valores de pertinência 0,75 e 0,70, respectivamente, sendo que o primeiro manteve-se nesta categoria com pertinência máxima, mas ocorreu um decréscimo para o valor 0,25. O cliente 13 findou a avaliação classificado com mobilidade Média, tendo pertinência máxima.

\section{DISCUSSÃO}

A enfermagem é uma profissão comprometida com a produção e gestão do cuidado nos diferentes contextos socioambientais e culturais em resposta às necessidades da pessoa, família e coletividade. Entre os deveres dos profissionais de enfermagem, destaca-se o Art. 45 do Código de ética - Prestar assistência de enfermagem livre de danos decorrentes de imperícia, negligência ou imprudência ${ }^{11}$. Assim, o enfermeiro e equipe de enfermagem possuem funções próprias que visam à qualidade de atendimento à pessoa hospitalizada, entre elas, as que possuem diagnóstico médico de DI. Entende-se que tais funções vão além do cumprimento das prescrições médicas, envolvem a avaliação da pessoa, definição de diagnósticos de enfermagem e implementação de cuidados para promoção de conforto e prevenção de agravos ${ }^{12,13}$, como as LP.

O DE mobilidade física prejudicada é definido como limitação do movimento independente e voluntário do corpo ou de uma ou mais extremidades ${ }^{8}$. A identificação deste diagnóstico nos participantes do estudo ocorreu a partir da presença das características definidoras: desconforto, redução na amplitude de movimentos, dificuldade para virar-se, para realizar movimentos lentos, e os fatores relacionados ansiedade, dor, índice de massa corporal acima dos $75 \%$ apropriados para a idade ${ }^{14}$.

O DE mobilidade no leito prejudicada caracteriza-se como limitação do movimento independente de uma posição para outra no leito ${ }^{8}$, foi identificado diante das características definidoras: capacidade prejudicada de reposicionar-se na cama, de mover-se, de virar-se de um lado para outro; além dos fatores relacionados: dor ${ }^{14}$.

A presença de lesões disseminadas ou localizadas na genitália e/ou face interna das coxas em 12 participantes dificultavam o movimento, fato agravado nos idosos e nos considerados acima do peso. Lembra-se que cinco participantes apresentaram índice de Massa Corporal alterada, compatível com obesidade em três adultos e sobrepeso em dois idosos.

Um estudo demonstrou maior frequência de LP nos clientes que apresentaram comprometimento prévio na pele, sendo observado que os cuidados com a pele são insuficientes quando as prioridades terapêuticas se sobrepõem as ações de prevenção de tal lesão. Tal fato revela que as instituições devem priorizar a elaboração e implementação de protocolos de prevenção para a melhoria da qualidade assistencial ${ }^{15}$.

Assim diante da integridade da pele previamente comprometida pelas lesões decorrentes das DI, destaca-se a vulnerabilidade aumentada para desenvolvimento de LP, que pode ser potencializada por fatores extrínsecos e intrínsecos. Sobre os extrínsecos, destacam-se uma leve pressão, fricção ou cisalhamento durante procedimentos rotineiros, como mudança de decúbito, troca de roupa de cama, uso de esfíngnomanômetro, garrotes, e ao toque durante transporte e/ou caminhada. A maceração pelo uso de fraldas e quantidade abundante de exsudato proveniente das lesões das DI. Entre outros fatores intrínsecos, destacam-se a fragilidade da epiderme, mobilidade prejudicada/ imobilidade, principalmente quando as lesões são mantidas expostas. Destaca-se ainda o uso de corticosteróides/imunossupressores, utilizados em altas doses para tratamento da DI, fato que pode levar a complicações como diabetes. Além disso, destaca-se a desnutrição, pela dificuldade de ingerir alimentos devido à presença de lesões na mucosa bucal ${ }^{1}$.

Sobre a utilização de curativos, lembra-se a necessidade de considerar a fisiopatologia das DI e especificidades das lesões, que podem ser agravadas caso seja utilizado curativo inadequado. Neste sentido, importa mencionar que devido a fisiopatologia das DI, os curativos realizados diferem dos curativos tradicionais, pois qualquer aderência no leito das lesões pode ampliar a área lesada e agravar a situação. Outro aspecto a ser ressaltado refere-se ao fato que se trata de um curativo de grande extensão que exige troca diária após higiene corporal, visando amenizar o odor proveniente das lesões/exsudato. Nesse sentido, destaca-se que o curativo com gaze vaselinada, preparado conforme protocolo proposto foi utilizado durante uma semana pelos 14 participantes do estudo, sendo constatada ausência de complicações e melhora significativa da mobilidade. Sobre isso menciona-se que devido as características fisiopatológicas das DI, a mínima aderência do curativo nas lesões, causa dor e aumento da área lesada, principalmente no momento da retirada. Os poucos trabalhos publicados referentes à aplicação de curativos nesta clientela 
específica ${ }^{5,16}$, não apresentam nível de evidência significativo, são estudos de caso abordando produtos que não impedem a aderência do curativo no leito das lesões ou curativos onerosos, que devido alto custo, possuem indicação de permanência por três/cinco ${ }^{16}$ dias, fato que impede a higiene corporal diária, causando desconforto/mal-estar, além de dificultar a recuperação.

\section{CONCLUSÃO}

Apesar da participação de 14 pessoas com DI, foi possível constatar o significativo impacto positivo do curativo com gaze vaselinada na mobilidade dos participantes, fato que ocorreu logo nas primeiras 24 horas após sua aplicação. Diante dos resultados obtidos, as evidências apontam para a veracidade da hipótese de que o curativo com gaze vaselinada, preparado conforme protocolo descrito neste estudo contribui para aumentar a mobilidade e consequentemente o conforto dos clientes com DI, o que impulsiona uma expectativa progressiva na prevenção de LP.

\section{REFERÊNCIAS}

1. Hanauer L, Azulay-Abulafia L, Azulay DR, Azulay RD. Dermatoses vesicobolhosas. In: Azulay RD, Azulay DR, Azulay-Abulafia L. Dermatologia. 7ạ ed. Rio de Janeiro: Guanabara Koogan; 2017. p. 930-8.

2. Brandão ES, Santos I, Lanzillotti RS. Reduction of pain in clients with autoimmune bullous dermatoses: evaluation by fuzzy logic. Online braz. j. nurs. 2016 [cited 2020 Jun03]; 15(4):675-82. Available from: http://www.objnursing.uff.br/index.php/nursing/article/view/5467/6611

3. Silva DAR, Bernardes AG. Pemphigus: cartography on the coordination of health policies. Ciênc. saúde coletiva [Internet]. 2018 [cited 2020 Jun 03]; 23(8):2631-40. DOI: https://doi.org/10.1590/1413-81232018238.14892016

4. Brandão ES, Santos I dos. Evidences related to the care of people with pemphigus vulgaris: a challenge to nursing. Online braz. j. nurs. 2013 [cited 2020 Jun 03]; 12(1):162-77. DOI: https://doi.org/10.5935/1676-4285.20133674

5. Soares HPL, Brandão ES, Tonole R. Primary bandages for people with pemphigus vulgaris lesions: an integrative literature review. Rev. Gaúcha Enferm. 2020 [cited 2020 Jun 03]; 41:e20190259. DOI: https://doi.org/10.1590/1983-1447.2020.20190259

6. National Pressure Ulcer Advisory Panel. National Pressure Ulcer Advisory Panel (NPUAP) announces a change in terminology from pressure ulcer to pressure injury and updates the stages of pressure injury. 2016. [cited 2020 Jun 03]. Available from: http://www.npuap.org/national-pressure-ulcer-advisory-panel-npuap-announces-a-change-in-terminology-from-pressureulcer-to-pressure-injury-and-updates-the-stages-of-pressure-injury/

7. Brandão ES, Santos I, Lanzillotti RS. Validation of a instrument to assess patients with skin conditions. Acta paul. enferm. 2013 [cited 2020 Jun04]; 26(5):460-6. DOI: http://dx.doi.org/10.1590/S0103-21002013000500009

8. NANDA International. Diagnósticos de enfermagem da NANDA-I: definições e classificação: 2018-2020. 11a ed. Porto Alegre: Artmed, 2018.

9. Souza SR, Azulay-Abulafia L, Nascimento LV. Validation of the commitment index of skin and mucous membranes of patients with pemphigus vulgaris for the clinical evaluation of patients with pemphigus vulgaris. An. bras. dermatol. 2011 [cited 2020 Jun 04]; 86(2):284-91. DOI: http://dx.doi.org/10.1590/\$0365-05962011000200012

10. Trillas E. Lofti A. Zadeh: on the man and his work. Scientia Iranica. 2011 [cited2020 Jun 04]; 18(3):574-579. DOI: https://doi.org/10.1016/j.scient.2011.05.001

11. Conselho Federal de Enfermagem. Resolução COFEN 564/2017. [cited 2020 Jun 04]. Available from: http://www.cofen.gov.br/resolucao-cofen-no-5642017_59145.html

12. Brandão ES, Santos I, Lanzillotti RS. Nursing care to comfort people with immunobullous dermatoses: evaluation by fuzzy logic. Rev. enferm. UERJ. 2018 [cited 2020 Jun 04]; 26e32877. DOI: https://doi.org/10.12957/reuerj.2018.32877

13. Brandão ES, Santos I. Theories of nursing in promotion of comfort in dermatology. Rev. enferm. UERJ. 2019 [cited 2020 Jun 03]; 27:e38330. DOI: http://dx.doi.org/10.12957/reuerj.2019.38330

14. Brandão ES, Santos I dos, Lanzillotti RS, Ferreira AM, Gamba MA, Azulay-Abulafia L. Nursing diagnoses in patients with immunebullous dermatosis. Rev. latinoam. enferm. [Internet]. 2016. [cited 2020 Jun 04]; 24(27e):27-66. DOI: http://dx.doi.org/10.1590/1518-8345.0424.2766

15. Mendonça PK, Loureiro MDR, Ferreira Júnior MA, Souza AS. Occurrence and risk factors for pressure injuries in intensive care centers. Rev. enferm. UFPE on line. 2018; [cited 2020 Jun 04]; 12(2):303-11. DOI: https://doi.org/10.5205/1981-8963v12i2a23251p303-311-2018

16. Costa MTFC, Oyafuso LKM, Costa IG, Gamba MA, Woo KY. The management of pemphigus ulcers: a challenge and learning opportunity for Brazilian nurses. World Council Enteros Ther J. 2016 [cited 2020 Jun 04]; 36(2): 14-27. Available from: http://docs.bvsalud.org/biblioref/2019/10/1021591/2016_p-015.pdf 